\title{
Utility optimization framework for a distributed traffic control of urban road networks
}

\author{
Tung Le ${ }^{a}$, Hai L. Vu ${ }^{b, *}$, Neil Walton ${ }^{c}$, Serge P. Hoogendoorn ${ }^{d}$, Péter Kovácse, \\ Rudesindo N. Queija ${ }^{\mathrm{e}}$ \\ a Intelligent Transport Systems Lab, Melbourne, Australia \\ ${ }^{\mathrm{b}}$ Institute of Transport Studies, Monash University, Australia \\ 'School of Mathematics, The University of Manchester, UK \\ d Delft University of Technology, The Netherlands \\ ${ }^{\mathrm{e}}$ Korteweg-de Vries Institute for Mathematics, University van Amsterdam, The Netherlands
}

\section{A R T I C L E I N F O}

\section{Article history:}

Received 25 May 2016

Revised 4 October 2017

Accepted 6 October 2017

Available online 20 October 2017

\section{Keywords:}

Distributed urban traffic control

Utility optimization framework

Gini coefficient

\footnotetext{
* Corresponding author.

E-mail address: hai.vu@monash.edu (H.L. Vu).
}

\section{A B S T R A C T}

Route choice behavior has been recognized as an important factor in the traffic management and control strategy design. State-of-the-art research in this field (e.g. anticipatory traffic control approaches) often formulates this as a bi-level optimization problem where users' reactions to changes in signal control are taken into account in the design of the control policies. Solving such a combined optimization problem is possible but difficult and complex which often involves the determination of the dynamic user equilibrium (UE). Nevertheless, anticipatory control inherently aims to optimize the signal control that anticipates or reacts to the users' route choice and thus is a reactive control. Furthermore, the approaches used in anticipatory control are based on the coupling between control and traffic assignment where the route choice behavior of road users is unrealistically assumed to be in equilibrium.

This paper proposes a novel utility based optimization framework to design a distributed control scheme based on a so-called BackPressure principle that is scalable and proactively influences the drivers' route choice behavior. In particular, as opposed to the well-studied anticipatory control and bi-level optimization, the proposed utility optimization formulation solves for a system optimal problem where the optimal turning fraction (as an output decision variable) is enforced as a desirable outcome. The optimal turning fractions would then be achieved in a practical deployment either by directly asking the users to follow the systems routing advice or by influencing, i.e. manipulating, the route choice decisions made by them so that the resulting turning fraction is (or is close to) the optimal value. Although drivers may not follow the computed optimal fractions as desire, there exist several ways, e.g. see Groot et al. (2015), to design appropriate incentives for drivers to follow the route guidance, and thus improve the compliance rate and overall journey experience for everyone inside the network. To this end, we demonstrate by numerical results that better network performance is achieved using the proposed framework even with a limited compliance from the users. Furthermore, we have formally proved that our proposed utility optimization based BackPressure scheme stabilizes the network for any feasible traffic demands thus maximizing network throughput. In addition, we show 
through simulation and numerical results that the new policy outperforms the original BackPressure-based distributed control scheme both in terms of network throughput and other congestion measures such as travel time.

(c) 2017 Elsevier Ltd. All rights reserved.

\section{Introduction}

Traffic congestion is a serious problem around the world and particularly in urban areas where both the population and traffic demand grow rapidly with limited or no room for a new infrastructure. The societal cost attributable to congestion is considerable due to the loss in productivity, reduction in safety and its negative impact on the environment. To date, traffic control is one of the oldest and most cost-effective solutions to combat traffic congestion. In particular, it has been reported that optimally designed traffic signals can lead to benefit-cost ratios as high as 40 to 1 (Fhwa, 2016).

Since its inception, traffic signals are used to control conflicting traffic flows at road intersections, and there have been many studies described in the transportation literature concerning the design and optimization of isolated or coordinated signals. Traffic signal controllers are usually described as either pre-timed or vehicle-actuated (Hamilton et al., 2013). Pretimed control (sometimes referred to as "fixed time plans") provides a repetitive cycle and its division of timing among the conflicting movements at the intersection. The timing is repeated again and again regardless of the traffic demand and can be optimized in isolation or in a coordinated manner (Gartner et al., 1975). Nevertheless, the fixed time plans degrade rapidly as traffic changes and they require regular update (or re-optimization) which is generally an expensive process (Papageorgiou et al., 2003).

Vehicle-actuated controls differ from pre-timed in that their signal timings are not fixed. Through the use of vehicles detectors (for example, inductive loops) this type of control optimizes signal timings on a cycle-to-cycle basis in real time. Practical systems of this type include SCOOT (Hunt et al., 1981); OPAC (Gartner, 1983); and the hierarchical scheme RHODES (Mirchandani and Head, 2001). Combinations of both the pre-timed and vehicle-actuated control also exist such as SCATS system (Lowrie, 1982).

\subsection{Complexity of network-wide control}

In the literature various approaches have been proposed to optimize the signal plans. Examples include mixed-integer linear programming problems for a single intersection (Dujardin et al., 2011) and for network of intersections (Gartner et al., 1975); rolling horizon optimization using dynamic programming, see Gartner (1983) and Mirchandani and Head (2001); store-and-forward models based on Model Predictive Control (MPC) optimization (Tettamanti and Varga, 2010; Le et al., 2013), or MPC optimization with non-linear prediction (Shu et al., 2011).

However, for a network with many intersections, the above centralized optimization methods result in exponential computational complexity and thus are not scalable. As noted in Papageorgiou et al. (2003), control strategies such as OPAC in Gartner (1983) and RHODES in Mirchandani and Head (2001) are not real-time feasible for more than one intersection. In fact, they actually became a distributed scheme via forced implementation at individual intersections that are heuristically coordinated over the network (Gartner et al., 2001).

On another note, it has been widely recognized that traffic signal control and drivers' route choice are the two main interdependent processes influencing traffic congestion. While the network operator aims to achieve system optimum by controlling traffic signals, road users adapt routes following their own personal objectives, e.g. by choosing the perceived fastest way to the destination. The work of Allsop (1974) was one of the first to point out the dependency between traffic control and drivers' route choice. Since then, there have been many proposed control frameworks in the literature (often referred to as anticipatory traffic control) to anticipate driver's route choice behavior while determining the optimal signal control. Particularly, the driver's route choice behavior has often been investigated via the existing mathematical framework of dynamic traffic assignment (DTA) since their reaction to changes in signal settings will cause changes in traffic flows resulting in a different traffic assignment. The modified traffic assignment would then require a new optimal signal settings and so on. Due to this interdependence between traffic control and traffic assignment, the usual approach to this coupled problem is to form a bi-level optimization and solve it either by iterative optimization and assignment procedures (van Zuylen and Taale, 2000; Taale, 2008) or by global optimization models (Chen and Ben-Akiva, 1998; Karoonsoontawong and Waller, 2010; Ukkusuri et al., 2013).

Although anticipatory control represents the state-of-the-art research in this field, it faces the same scalability problem in terms of complexity. Recent attempt has been made by Rinaldi and Tampère (2015) toward a distributed version of the anticipatory scheme by subdividing the original optimization problem into smaller, more tractable sub-problems while aiming to achieve global optimality (Rinaldi and Tampère, 2015). Nevertheless, the approaches used in anticipatory control are based on the coupling between control and traffic assignment where the route choice behavior of road users is assumed to be in equilibrium. Such equilibrium is typically only applicable in the context of a long term (e.g. strategic planning) application, and is not necessary true in the instantaneous, real-time traffic actuated dynamic signal control scenarios. Furthermore, 
solving this user equilibrium (UE) via the DTA framework with realistic traffic conditions (e.g. stochastic) is complex. More importantly, anticipatory control inherently aims to optimize the signal control that adapts to the users' route choice. The system then tends to converge to UE which is subject to the aggregate effect of users' route choice. However, the UE, even if it is optimized by anticipatory frameworks, may not present the best possible throughput the system may achieve. For instant, we may have better throughput simply if the users choose their routes differently. In reality, the network and its road users might not even be in equilibrium. In addition, gathering the demand information where each traveler has his own route choice preference presents further difficulties.

In sum, the complexity and the suboptimal cause by users' route choice behavior are the two problems that we will tackle in this paper. To this end, we will proposed a novel methodology underpinned by a mathematical utility optimization framework solving for a system optimal problem to design a distributed control scheme that is scalable and actively influences the drivers' route choice behavior. Our proposed control scheme will include the route choice responses by (a) introducing the set $\mathcal{H}$, which is the set of all achievable expected turning fractions, into the optimization constraints and (b) introducing the compliance probability into the simulation study, i.e. users might chose not to follow the systems advice. The former enables us to enforce the optimal turning fraction as a desirable outcome jointly with the signal control. While the latter models the users response to advice via a compliance probability that users might choose not to follow the route recommendation. Remarkably, as we show later via numerical results, better network performance is achieved using the proposed framework even with a limited compliance from the users. This distributed framework enables us to decouple the optimal control scheme from the complex DTA UE formulation, but still take into account the users' route choice behavior and thus tackle both the complexity and the UE issues.

\subsection{State-of-the-art of distributed control}

Below we review the related literature in the area of distributed (or decentralized) control before detailing the approach and contributions of this paper. Despite the obvious advantages in scalability and ease in implementation, distributed control strategies are not yet practical in the context of urban traffic management. It is because a distributed scheme will have to rely on detailed local information collected at the intersection in real-time to be effective. distributed Such information can be either (a) the expected number of vehicles to enter the intersection during the next cycle, or (b) the difference in traffic load on the road leading into the intersection and those leading out. These approaches are now deployable in practice thanks to emerging technologies, such as smart detectors, cameras and wireless communication enabling better access to real-time traffic data.

Early work of Smith (1980), a so-called $P_{0}$ local control policy and its variants (Smith, 2011) belong to the former (approach (a)). Follows by Lämmer and Helbing (2008) where the authors extended further the $P_{0}$ policy to include the switching cost between phases. A heuristic mechanism was proposed in Lämmer and Helbing (2010) to prevent possible instability but there remains no formal proof of stability for such distributed control strategy. Note that the notion of stability will be formally defined later in Section 5.

The latter (approach (b)) include work by Wunderlich et al. (2008), Varaiya (2013), Wongpiromsarn et al. (2012) and Le et al. (2015) all of which were based on a so-called max weight or back pressure (refer to as BackPressure) algorithm (Tassiulas and Ephremides, 1992) originally developed for packet scheduling in wireless data networks. The BackPressure based distributed control strategy is very attractive because it can ensure maximum possible network throughput without $a$ priori knowledge of the traffic demand. More importantly it has the added benefit of provable stability. In addition, J. de Gier and Rojas (2011) report that for a typical system, the control strategy that uses congestion observed on both upstream and down stream links (i.e. BackPressure type) is more efficient and reliable in term of travel times than that informed by the congestion on the upstream link only (i.e. approach (a)). Further the backpressure policy can incorporate and route multicommodity flows provided a separate queue is maintained for each commodity, such a system was first proposed by Tassiulas and Ephremides (1992). With some loss of stability, can be adapted to traffic control mechanisms which are agnostic to the commodities used, see Bui et al. (2009) and Zaidi et al. (2016).

The BackPressure mechanism, however, has a drawback as it can lead to a large queue build up at the upstream of a route between an origin-destination (OD) pair (Stolyar, 2011) resulting in traffic spatial heterogeneity. This is undesirable because larger upstream queues will increase the spatial variation in the density across the network which in turns reduces a so-called "level-of-service" in the network, for more details see Hoogendoorn et al. (2013). In the other words, the impact of congestion worsens when the heterogeneity of link densities or queues increases. There exist mitigation techniques for this problem in the literature of wireless networking, but for a traffic control scenario, they would require the knowledge of individual vehicle's destination (such as in Bui et al. (2009)) which is clearly not feasible.

Given the advantages of the BackPressure based traffic control, our methodology in this paper is to form a novel utility optimization framework with an appropriately chosen utility function that can reduce the traffic spatial heterogeneity while maximizing possible throughput just as in the original BackPressure algorithm. To this end, we propose the use of a so-called Gini-index (Foster and Sen, 1973) as an indication of network density inequality where a Gini-index value of zero presents a perfect equality while higher value presents greater density deviation. This index will then serve as a measure for traffic spatial heterogeneity and will be jointly minimized in the above utility optimization framework. Furthermore the proposed framework will directly influence drivers' route choice behavior by giving out local route guidance advice, for instance which next road an user might want to take, without assuming that the user will actually comply with the advice. If the driver does 
Table 1

Comparison between our proposed policy and other policies.

\begin{tabular}{|c|c|c|c|c|}
\hline Works & $\begin{array}{l}\text { Centralized or } \\
\text { distributed }\end{array}$ & $\begin{array}{l}\text { Proven stability } \\
\text { (optimal throughput) }\end{array}$ & $\begin{array}{l}\text { Anticipate users' } \\
\text { route choice }\end{array}$ & $\begin{array}{l}\text { Influence users' route } \\
\text { choice }\end{array}$ \\
\hline (Tettamanti and Varga, 2010) & Centralized & Yes & No & No \\
\hline (Le et al., 2013) & Centralized & Yes & No & Yes \\
\hline (Taale, 2008; Ukkusuri et al., 2013) & Centralized & Yes & Yes & No \\
\hline (Smith, 1980; 2011; Smith et al., 2015) & Distributed & $\begin{array}{l}\text { Yes, but not for general } \\
\text { networks }\end{array}$ & Yes & No \\
\hline $\begin{array}{l}\text { (Wunderlich et al., 2008; Varaiya, 2013; } \\
\text { Wongpiromsarn et al., 2012; Le et al., 2015) }\end{array}$ & Distributed & $\begin{array}{l}\text { Yes, for general } \\
\text { networks }\end{array}$ & No & No \\
\hline Our proposed framework & Distributed & $\begin{array}{l}\text { Yes, for general } \\
\text { networks }\end{array}$ & No & Yes \\
\hline
\end{tabular}

indeed follow the advice and enters link that was not part of the original planned route, then she will need to recalculate the route to the destination based on her own preference. From the network control's perspective, these advertised local turning advices are seen as a desirable route choice of the road users which helps to reduce the overall congestion in the network. The signal control and traffic assignment (or turning fractions) are then jointly optimized in our utility framework which is in a stark contrast to the anticipatory control scheme where bi-level optimization coupling with dynamic traffic assignment problems are solved iteratively. In other words, the traffic signal control and desirable users' behavior are jointly optimized in a single optimization which greatly reduces the complexity of the problem. Beside we do not need to involve the step of finding the user equilibrium via the DTA formulation based on a particular users' route choice model.

\subsection{Research contributions and paper outline}

The idea of jointly optimizing the traffic signal control and desirable route choice, that is to influence rather than follow drivers' behavior, was proposed in our previous work (Le et al., 2013) but for a centralized signal control using model predictive control (MPC). Recent work by Smith et al. (2015) and Groot et al. (2015) follow a similar approach, and the latter also provides several ways to design appropriate incentive for drivers to follow the route guidance, and thus improve the compliance rate and overall journey experience for everyone inside the network. In this paper, we will explore this concept for a distributed control scheme based on the BackPressure principle. The study of design and giving incentive to encourage and improve users' compliance is out of the scope of this paper and will be investigated in a separate work. Nevertheless, we show via numerical examples that the BackPressure derived from the proposed utility optimization framework outperforms the original BackPressure even with a limited user compliance (Wunderlich et al., 2008; Varaiya, 2013; Wongpiromsarn et al., 2012) both in terms of throughput and congestion metrics. See Table 1 for the summary comparison between our proposed framework and other frameworks in the literature.

The main contributions in this paper include

- a novel utility optimization framework together with the use of the Gini-index as an utility function that indicates the level of network spatial density inequality,

- a novel control policy that is scalable and actively influence on the users' route choice that potentially outperform other distributed anticipatory control policies.

- a new distributed optimal traffic control strategy that combines signal phasing and route guidance for urban traffic networks based on the BackPressure mechanism's principle,

- a formal mathematical proof that the proposed policy is stable, i.e. achieving a maximum possible network throughput whenever the demand is feasible,

- a control strategy that does not require knowledge about the user's route choice or traffic demand while still be able to maximize the network throughput and improve on congestion measure, and

- results to demonstrate under different level of compliance that (i) the performance improves significantly even with limited user compliance, (ii) a relatively small percentage of compliance (e.g. 10\%) is enough to gain significant benefits.

The remainder of this paper is organized as follows. We describe the notations and network dynamics model before describing our proposed utility optimization based BackPressure policy in Section 2. The main results for stability of our policy are then provided in Section 3. For readability most of the mathematical details and derivations are listed in the Appendices of the paper. Section 4 presents a simple case study where a control policy with users' route choice influence could outperform other distributed anticipatory policies. Section 5 presents the simulation results and numerical comparison of our scheme with the existing BackPressure policy. Finally, Section 6 concludes the paper. 


\section{Methodology: the utility optimization framework}

\subsection{Notation and network description}

Consider a road traffic network involving a collection of junctions (or intersections) $\mathcal{J}$. Each junction $j \in \mathcal{J}$ consists of a number of in-roads $\mathcal{I}_{j}$ representing an incoming road to that junction. Note that $\mathcal{I}_{j}$ are disjoint and let $\mathcal{I}=\cup_{j \in \mathcal{J}} \mathcal{I}_{j}$.

Each junction may serve different combinations of in-roads simultaneously by the mean of controlling traffic signals. We call such combination a service phase. A service phase of junction $j$ is presented by a vector $\Psi=\left(\psi_{i}: i \in \mathcal{I}_{j}\right)$ where $\psi_{i}=c_{i}$ if in-road $i$ belongs to service phase $\Psi$ and $\psi_{i}=0$ otherwise, and $c_{i}$ is the capacity of in-road $i$. Note that in-road capacity $c_{i}$ is the expected number of cars to be served during a traffic signal cycle given that the whole cycle is allocated as a green signal to the in-road $i$ and that $i$ is not empty at any time point during the cycle. Let $\mathcal{S}_{j}$ denote the set of phases at junction $j$.

Furthermore, let $\mathcal{L}$ denote the set of links representing the traffic movements at a junction of the traffic network. We denote $i i^{\prime} \in \mathcal{L}$ if there exist $i \in \mathcal{I}_{j}$ and $i^{\prime} \in \mathcal{I}_{j^{\prime}}$ such that it is possible for vehicles to be served at $i$ to next join at $i^{\prime}$.

We assume all junctions share a common traffic signal cycle length $T$. Then we can represent time as discrete $t=0,1, \ldots$ and consider a slotted time model where each time slot corresponding to a traffic light cycle. Control decisions in our policy are then made at the beginning of each time slot similar to the policies previously proposed in Lämmer and Helbing (2010), Varaiya (2013) and Smith (1980).

\subsection{Network dynamics model}

Let $q_{i}(t)$ denote the number of vehicles at in-road $i \in \mathcal{I}$ at the beginning of time slot $t$. We assume the number of vehicle at an in-road can grow unbounded. We define a vector $Q(t)=\left(q_{i}(t): i \in \mathcal{I}\right)$. Let $p_{\Psi}^{j}(t)$ denote the fraction of the traffic cycle at time slot $t$ at junction $j$ which is devoted to service phase $\Psi$. We define the control vector as $P_{j}=\left(p_{\Psi}^{j}: \Psi \in \mathcal{S}_{j}\right)$. Note that $P_{j}$ are disjoint and let $P=\cup_{j \in \mathcal{J}} P_{j}$. For any policy, we define a feasible control set as:

$$
\Pi_{j}=\left\{P_{j}: \sum_{\Psi \in \mathcal{S}_{j}} p_{\Psi}^{j} \leq 1, p_{\Psi}^{j}>0\right\} \forall j \in \mathcal{J} .
$$

For any in-road $i \in \mathcal{I}$, the expected number of vehicles leaving during service phase $\Psi$ is $\psi_{i} p_{\Psi}^{j}(t)$, provided the in-road is not emptied. Accordingly if we let the random variable $s_{i}(t)$ be the potential number of cars served from in-road $i$ at junction $j$ in traffic cycle $t$, the mean of $s_{i}(t)$ is given as

$$
\mathbb{E}\left[s_{i}(t) \mid q_{i}(t)\right]=\sum_{\Psi \in \mathcal{S}_{j}} \psi_{i} p_{\Psi}^{j}(t) .
$$

In other words, the variable $s_{i}(t)$ gives the offered load on in-road $i$, which is the number of vehicles served if there is an indefinite queue of vehicles. If the number of vehicles in in-road $i$ is not high enough to be served during the right-ofway (or green phase) service time, the actual number of vehicles leaving $i$ is $q_{i}(t)$. Hence, the number of vehicles served at in-road $i$ is a random variable defined by:

$$
f_{i}(t)=\min \left(s_{i}(t), q_{i}(t)\right)
$$

For any $i i^{\prime} \in \mathcal{L}$, let $\theta_{i i^{\prime}}(t)$ be the proportional of cars served at in-road $i$ that subsequently join in-road $i^{\prime}$ at time $t$. We assume that cars within an in-road are homogeneous in the sense that each car at the junction has the same likelihood of joining each subsequent junction. We denote the expectation of $\theta_{i i^{\prime}}(t)$ by $\bar{\theta}_{i i^{\prime}}(t)$, and write the expectation of turning fractions in a vector form $\bar{\Theta}_{j}(t)=\left(\bar{\theta}_{i i^{\prime}}(t): i \in \mathcal{J}, i i^{\prime} \in \mathcal{L}\right)$. In other words

$$
\mathbb{E}\left[\theta_{i i^{\prime}}(t) \mid s_{i}(t), q_{i}(t)\right]=\bar{\theta}_{i i^{\prime}}(t)
$$

Note that $\bar{\Theta}_{j}$ are disjoint and let $\bar{\Theta}=\cup_{j \in \mathcal{J}} \bar{\Theta}_{j}$.

This expected turning fraction, which is known or can be measured, is an aggregated result of individual route choices and can be influenced by systems such as advanced travel information system (ATIS). Assuming a certain route guidance compliance, we define $\mathcal{H}_{j}$ as the set of all achievable expected turning fractions of junction $j$. The set of turning fractions is a closed set has the property that if two turning fractions are achievable at a junction, then a combination of these turning fraction is also achievable. More formally, we assume that if $\theta, \phi \in \mathcal{H}_{j}$ and are such that, for some $i, \theta_{i^{\prime} i^{\prime \prime}}=\phi_{i^{\prime} i^{\prime \prime}}$ for all $i \neq i^{\prime}$ then for $\alpha \in(0,1), \alpha \theta+(1-\alpha) \phi \in \mathcal{H}_{j}$.

We further assume that this set is constant and will not be altered by the queue lengths observed by cars within the network. Thus $f_{i}(t) \theta_{i i^{\prime}}(t)$ is the number of cars that leave inroad $i$ and, next, join inroad $i^{\prime}$. We let $A(t)=\left(a_{i}(t): i \in \mathcal{I}\right) \in \mathbb{Z}_{+}^{\mathcal{I}}$ where $a_{i}(t)$ which is a stochastic quantity denotes the number of external arrivals at in-road $i$ at time $t$. The $a_{i}(t)$ assumed to be independent identically distributed over times $t$ with finite mean and variance. 
Given a service policy $\left\{P_{j}(t), \Theta_{j}(t): j \in \mathcal{J}\right\}_{t=0}^{\infty}$, below we can define the dynamics of our network as a random queueing process for the in-road $i$ of junction $j$, as follows,

$$
q_{i}(t+1)=q_{i}(t)-f_{i}(t)+a_{i}(t)+\sum_{i^{\prime} i^{\prime} i \in \mathcal{L}} f_{i^{\prime}}(t) \theta_{i^{\prime} i}(t)
$$

for $P_{j}(t) \in \Pi_{j}$ and $\bar{\Theta}_{j}(t) \in \mathcal{H}_{j}$.

\subsection{Utility optimization based BackPressure (U-BP) control policy}

We first introduce an utility function $g_{j}\left(P_{j}(t), \Theta_{j}(t)\right)$ that is bounded such as $0 \leq g_{j}\left(P_{j}(t), \bar{\Theta}_{j}(t)\right) \leq G$ for all $t \in \mathbb{R}^{+}$. One such function is a so-called Gini index function (Foster and Sen, 1973) which is always bounded between [0, 1], and can be used as a measure of the inequality (or spatial heterogeneity) of traffic within the urban traffic network.

Given the advantages of the BackPressure algorithm for traffic control discussed earlier in Section 1, the idea here is to form a novel utility optimization problem that jointly stabilizes the network (i.e. maximizes possible network throughput as in BackPressure algorithm) and at the same time minimizes the chosen utility function (i.e. reduce traffic spatial heterogeneity). Note that the utility function is not restricted to the aggregate of individual traveling gain, it is rather the generalized cost from network perspective. The proposed policy is described as follows.

\section{The U-BP control policy:}

1. At the beginning of each traffic cycle and for each junction $j \in \mathcal{J}$, we define the weight associated with each service phase at the junction as a function of the queue sizes $Q(t)$ and some initial expected turning probabilities $\bar{\theta}_{i i^{\prime}}(t)$

$$
w_{\Psi}(Q(t))=\sum_{i \in \mathcal{I}_{j}} \psi_{i}\left(q_{i}(t)-\sum_{i^{\prime}: i i^{\prime} \in \mathcal{L}} \bar{\theta}_{i i^{\prime}}(t) q_{i^{\prime}}(t)\right) .
$$

2. Given these weights, determine the traffic signal phase vector $P_{j}(t)$ and the expected turning fraction vector $\bar{\Theta}_{j}(t)$ for each junction $j \in \mathcal{J}$ that are the solution of the following minimization problem

$$
\begin{array}{ll}
\underset{P_{j}(t), \bar{\Theta}_{j}(t)}{\operatorname{minimize}} & \mathbb{E}\left[g_{j}\left(P_{j}(t), \bar{\Theta}_{j}(t)\right) \mid Q(t)\right]-\sum_{\Psi \in \mathcal{S}_{j}} p_{\Psi}^{j}(t) w_{\Psi}(Q(t)) \\
\text { subject to } & P_{j}(t) \in \Pi_{j} \quad \text { and } \quad \bar{\Theta}_{j}(t) \in \mathcal{H}_{j}
\end{array}
$$

3. Set the control signals at the junction according to $P_{j}(t)$ while aim to achieve the optimal expected turning fraction $\bar{\Theta}_{j}(t)$ via giving route guidance advices to road users.

The weights defined in (6) are used in the BackPressure policy as given by Tassiulas and Ephremides (1992). They can be viewed as a "pressure" a queue places on downstream queues, which is given by the weighted mean of the differences of the queue sizes. The larger the weight associate with a phase, the more important it is to serve the in-roads with green lights during that phase.

Here the optimisation problem (7) need not be linear. As queue sizes get large, the problem will be linear and thus can be solved. Provided the function $g_{j}$ has a sub-gradient, then gradient descent methods will find a local-optimum. A global optimum is not required by the algorithm, since these notations local and global will coincide asymptotically and will be close during busy periods. Specifically, due to compactness of region and convergence to linear function, the values of the local minima will converge to the global optimum. Notice that in contrast to BackPressure policies which always serve the phase associated with the highest weight, the proposed policy balances between higher weights and a larger inequality (or spatial heterogeneity). The latter is due to the "needs" to increase queue size closed to the origin (source) in the BackPressure policy. Here giving route guidance advice is a practical mechanism that we use to achieve the desired expected turning fraction at the junction. We will elaborate it in more details in Section 5 where a compliance probability is introduced to reflect the fact that road users might not comply to the advice.

Also note that the utility measures traffic queue locally and thus the policy can be implemented in a distributed way, after each junction communicates queue sizes with its upstream in-roads, the phases and turning fractions can be calculated. This decentralization has numerous advantages: it is computationally inexpensive, it does not require centralized aggregation of information and thus is easier to implement, and it increases the road networks robustness to failures.

\section{Theoretical results - stability of the U-BP control policy}

Below we will formally define and prove the stability property of the proposed U-BP control scheme.

\subsection{Assumptions}

The number of cars that can be served from any in-road within a traffic cycle is bounded $S_{\max }=\max _{t \in \mathbb{Z}_{+}, i \in \mathcal{I}} s_{i}(t)<\infty$. The arrival is bounded $A_{\max }=\max _{t \in \mathbb{Z}_{+}, i \in \mathcal{I}} a_{i}(t)<\infty$. For any in-road $i$, the number of in-road $i^{\prime}$ such that it is possible for vehicles travel from $i^{\prime}$ to $i$ is bounded $D_{\max }=\max _{i \in \mathcal{I}} \operatorname{card}\left(i^{\prime}: i^{\prime} i \in \mathcal{L}\right)<\infty$. 


\subsection{Queueing stability}

We define the total queue size of the road network to be

$$
q^{\Sigma}(t)=\sum_{i \in \mathcal{I}} q_{i}(t)
$$

So $q^{\Sigma}(t)$ gives the total number of cars within the road network. We say that a policy is stable if the long run number of cars in the queueing network is finite, in particular,

$$
\lim _{T \rightarrow \infty} \mathbb{E}\left[\frac{1}{T} \sum_{t=1}^{T} q^{\Sigma}(t)\right]<\infty
$$

We note that if the queue size process was a Markov chain then definition of stable would be equivalent to the definition of positive recurrence for that Markov chain. However, the process that we will define need not be a Markov chain hence we use the above definition.

\subsection{Stability region}

We consider the set of arrival rates $\bar{A}=\left(\bar{a}_{i} \geq 0: i \in \mathcal{I}\right)$, for which there exists a positive vectors $\bar{P}=\left(\bar{p}_{\Psi}^{j}: \Psi \in \mathcal{S}_{j}, j \in \mathcal{J}\right) \in$ $\Pi$ where $\Pi=\cup_{j \in \mathcal{J}} \Pi_{j}, \bar{F}=\left(\bar{f}_{i}: i \in \mathcal{I}\right)$ and $\bar{\Theta}=\left(\bar{\theta}_{i i^{\prime}}: i i^{\prime} \in \mathcal{L}\right) \in \mathcal{H}$ where $\mathcal{H}=\cup_{j \in \mathcal{J}} \mathcal{H}_{j}$, namely the green time proportions, the departure rates, and the turning fractions respectively, that satisfy the following constraints

$$
\begin{aligned}
& \bar{a}_{i}+\sum_{i^{\prime}: i^{\prime} i \in \mathcal{L}} \bar{f}_{i^{\prime}} \bar{\theta}_{i^{\prime} i} \leq \bar{f}_{i}, \quad \forall j \in \mathcal{J}, \quad \forall i \in \mathcal{I}_{j} \\
& \bar{f}_{i} \leq \sum_{\Psi \in \mathcal{S}_{j}} \bar{p}_{\Psi}^{j} \psi_{i}, \quad \forall i \in \mathcal{I},
\end{aligned}
$$

and denote this set by $\mathcal{A}$. This set is clearly convex by the definition of $\Pi_{j}$ and $\mathcal{H}_{j}$. We let $\mathcal{A}^{\circ}$ denote the interior of $\mathcal{A}$ where the above inequalities in (10) and (11) are strict. We call $\mathcal{A}$ the stability region of the system as verified by the following proposition and our main theorem. Informally, they state that for any demand outside the stability region there is no schedule that would prevent the queue sizes to grow infinitely in the long run, whereas if $\bar{A} \in \mathcal{A}^{\circ}$ the BackPressure policy stabilizes the system.

Proposition 1. Given that the arrivals at each time, $\{A(t)\}_{t=1}^{\infty}$, are independent identically distributed random variables with expectation $\bar{A}$, then it follows that if $\bar{A} \notin \mathcal{A}$ then, for any policy, the queue sizes $\{Q(t)\}_{t=0}^{\infty}$ are unstable.

Proof. If $\bar{A} \notin \mathcal{A}$ then there exists an $\epsilon>0$ where, for any vectors $\bar{P}=\left(\bar{p}_{\Psi}^{j}: \Psi \in \mathcal{S}_{j}, j \in \mathcal{J}\right) \in \Pi, \bar{F}=\left(\bar{f}_{i}: i \in \mathcal{I}\right)$ and $\bar{\Theta}=\left(\bar{\theta}_{i i^{\prime}}\right.$ : $\left.i, i^{\prime} \in \mathcal{I}\right) \in \mathcal{H}$ satisfying (11), there exists an in-road $i$ such that

$$
\bar{a}_{i}-\bar{f}_{i}+\sum_{i^{\prime}: i^{\prime} i \in \mathcal{L}} \bar{f}_{i^{\prime}} \bar{\theta}_{i^{\prime} i}>\epsilon .
$$
limits

For any policy $\left\{P_{j}(t), \bar{\Theta}_{j}(t): j \in \mathcal{J}\right\}_{t=0}^{\infty}$ taking a suitable subsequence if necessary we have the existence of the following

$$
\begin{gathered}
\bar{f}_{i}=\lim _{T \rightarrow \infty} \frac{1}{T} \sum_{t=1}^{T} f_{i}(t), \quad \bar{\eta}_{i i^{\prime}}=\lim _{T \rightarrow \infty} \frac{1}{T} \sum_{t=1}^{T} f_{i}(t) \theta_{i i^{\prime}}(t), \\
\bar{\theta}_{i i^{\prime}}=\lim _{T \rightarrow \infty} \frac{1}{T} \sum_{t=1}^{T} \frac{f_{i}(t)}{\sum_{s \leq T} f_{i}(s)} \theta_{i i^{\prime}}(t)
\end{gathered}
$$

Note that

$$
\bar{\eta}_{i i^{\prime}}=\lim _{T \rightarrow \infty}\left[\frac{\sum_{t=1}^{T} f_{i}(t)}{T} \sum_{t=1}^{T} \frac{f_{i}(t)}{\sum_{s \leq T} f_{i}(s)} \theta_{i i^{\prime}}(t)\right]=\bar{f}_{i} \bar{\theta}_{i i^{\prime}}
$$

Further we claim that $\bar{\Theta}=\left(\bar{\theta}_{i i^{\prime}}: i i^{\prime} \in \mathcal{L}\right) \in \mathcal{H}$. This holds since for each $i$ the vector $\Theta_{i}^{T}=\left(\theta_{i i^{\prime}}^{T}: i i^{\prime} \in \mathcal{L}\right)$ whose components are given by

$$
\theta_{i i^{\prime}}^{T}=\sum_{t=1}^{T} \frac{f_{i}(t)}{\sum_{s \leq T} f_{i}(s)} \theta_{i i^{\prime}}(t)
$$

is a convex combination of junction $i$ turning fractions in $\mathcal{H}$. Thus the limit of these $\left(\bar{\theta}_{i i^{\prime}}: i i^{\prime} \in \mathcal{L}\right)$ belongs to $\mathcal{H}$. 
It is clear that $\bar{f}$ satisfies constraint (11) (being the average of terms satisfying this constraint); However since $\bar{A} \notin \mathcal{A}$ it must be that (12) occurs for some $i$. If we combine this with the fact, that the long run in-road queue size must converge to the average arrival minus the average departures, we get

$$
\lim _{t \rightarrow \infty} \frac{q^{\Sigma}(t)}{t}=\sum_{i \in \mathcal{I}}\left(\bar{a}_{i}+\sum_{i^{\prime}: i^{\prime} i \in \mathcal{L}} \bar{f}_{i^{\prime}} \bar{\theta}_{i^{\prime} i}-\bar{f}_{i}\right)>\epsilon
$$

In the above inequality, we note that each term in the summation is positive since it is the limit of positive queue sizes and one of those terms in greater than $\epsilon$ by (12). Thus we see that for any policy there exists a finite $\vartheta$ such that for all $t>\vartheta$,

$$
q^{\Sigma}(t)>t \epsilon
$$

thus,

$$
\lim _{T \rightarrow \infty} \frac{1}{T} \sum_{t=1}^{T} q^{\Sigma}(t) \geq \lim _{T \rightarrow \infty} \frac{1}{T} \sum_{t=\vartheta}^{T} \epsilon t=\infty .
$$

So, taking expectations, we see that the network must be unstable as stated in Proposition 1, since (9) cannot hold as

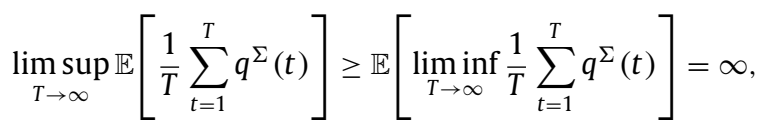

where we applied Fatou's Lemma (Ahmed and Ahmed, 2006).

\subsection{Main theorem}

Our main theorem considers the stability of the network. Namely, we prove that under the proposed signal control policy the system is stable for any feasible demand. As Proposition 1 shows this is the most any policy can provide.

Theorem 1. If $\bar{A} \in \mathcal{A}^{\circ}$, i.e. there exists an $\epsilon>0$ such that $\bar{A}+\epsilon \mathbf{1} \in$ Athen by applying the U-BP control policy defined in Section 2.32.3 the long run average queue sizes of in-roads are bounded as

$$
\lim _{\tau \rightarrow \infty} \mathbb{E}\left[\frac{1}{\tau} \sum_{t=0}^{\tau-1} q^{\Sigma}(t)\right] \leq \frac{|\mathcal{I}| K+\| \mathcal{J} \mid G}{\epsilon}
$$

where

$$
K=\frac{1}{2}\left(A_{\max }+D_{\max } S_{\max }\right)^{2}+\frac{3 S_{\max }^{2}}{2} .
$$

and thus the policy is stable.

The proof of Theorem 1 is given in Appendix A and follows a somewhat standard Lyapunov analysis for BackPressure policy, e.g. see Tassiulas and Ephremides (1992). Nevertheless, there are important features that must be considered in this particular extension. Specifically the non-standard turning fractions are part of the control mechanism and must be considered in the definition of the stability region. Also the bounded error introduced by the $g_{j}$ function in (7) must be dealt with in the proof.

From the Little's Theorem, the average travel time, $\bar{D}$, can be derived as followed

$$
\bar{D}=\frac{\lim _{\tau \rightarrow \infty} \mathbb{E}\left[\frac{1}{\tau} \sum_{t=0}^{\tau-1} q^{\Sigma}(t)\right]}{\sum_{i \in \mathcal{I}} \bar{a}_{i}} \leq \frac{|\mathcal{I}| K|\mathcal{J}| G}{\epsilon \sum_{i \in \mathcal{I}} \bar{a}_{i}} .
$$

\section{A simple scenario with route choice model}

This section illustrates the benefit of actively influence users' route choice through a simple scenario. The end results will show that a control policy that actively influence users' route choice could result in better overall travel delay than the control policy that does not influence on users' route choice, say Proportional policy (Smith, 2011) and BackPressure policy (Wunderlich et al., 2008; Varaiya, 2013; Wongpiromsarn et al., 2012). To make the terminology clear when route choice model is involved, all control policies in someway influence on users' route choice through changing the network states, however, a policy that actively influence users' route choice rather changes the decision making criteria. For example, it is common to assume that users always choose the fastest route. A policy that influences users' route choice would have the effect that sometime users may choose the longer route either unconsciously based on the information given to them or consciously for a greater good. We will have quantitative analysis for this effect in the following example. 


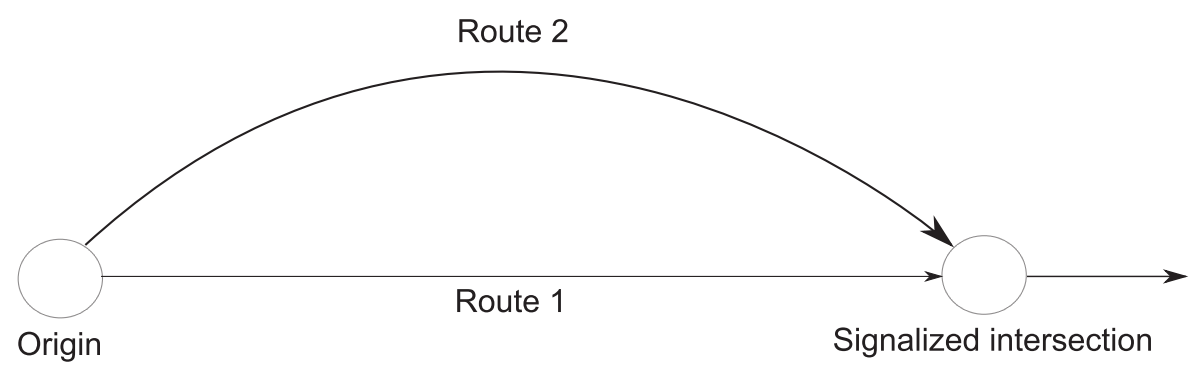

Fig. 1. A simple network.

We consider a simple network topology in Fig. 1 with a single OD pair, one intersection and two routes. Upon departure the users have a choice of what route to follow which is similar to turning fraction in general networks. Route 1 has smaller road and short distance, so the delay to travel on route 1 is modeled as

$$
b_{1}=\frac{Q_{1}}{s_{1} G_{1}}=\frac{Q_{1}}{G_{1}}
$$

where $Q_{1}$ is the number of cars waiting to be served at the intersection followed route $1, s_{1}$ is the saturated flow going through the intersection from route 1 and $s_{1}=1, G_{1}$ is the green time fraction allocated for route 1 .

Route 2 has bigger road but longer distance, so the delay to travel on route 2 is modeled as

$$
b_{2}=d+\frac{Q_{2}}{s_{2} G_{2}}=d+\frac{Q_{2}}{2 G_{2}}
$$

where $d$ is a constant which represents the free flow travel time, $Q_{2}$ is the number of cars waiting to be served at the intersection followed route $2, s_{2}$ is the saturated flow going through the intersection from route 2 and $s_{2}=2, G_{2}$ is the green time fraction allocated for route 2 .

Let $\lambda_{1}, \lambda_{2}$ are the arrival at each route. There is a constant demand $a$ for this scenario so

$$
\lambda_{1}+\lambda_{2}=a \text {. }
$$

The signalized intersection operates on fixed cycle time. Depend on the control policy, the intersection will allocate the green signal to either route 1 or route 2 at any point in time, hence we have the constraint

$$
G_{1}+G_{2}=1 \text {. }
$$

For this network, the maximum throughput is 2 when all vehicles travel on route 2 and all green time is allocated for route 2 . Similarly, the minimum throughput is 1 when all vehicle travel on route 1 and all green time is allocated for route 1. Therefore, the network is always unstable if $a>2$ and the control problem becomes trivial if $a<1$. So we only focus on the case $1 \leq a \leq 2$.

The queue dynamics are modelled as follows

$$
\begin{aligned}
& \frac{d Q_{1}}{d t}=\lambda_{1}-s_{1} G_{1}=\lambda_{1}-G_{1} \\
& \frac{d Q_{2}}{d t}=\lambda_{2}-s_{2} G_{2}=\lambda_{2}-2 G_{2}
\end{aligned}
$$

In the rest of this section, we will analyze this system at the user equilibrium with Proportional policy, BackPressure policy and the BackPressure policy with users' route choice influence.

\subsection{Proportional policy}

The Proportional policy allocates green time proportional to the queues. Thus, we have

$$
\frac{G_{1}}{Q_{1}}=\frac{G_{2}}{Q_{2}} .
$$

The equilibrium state of Proportional policy

$$
\begin{aligned}
& b_{1}=b_{2} \\
& \frac{d Q_{1}}{d t}=0 \\
& \frac{d Q_{2}}{d t}=0 \\
& \frac{G_{1}}{Q_{1}}=\frac{G_{2}}{Q_{2}}
\end{aligned}
$$




$$
\begin{aligned}
& G_{1}+G_{2}=1 \\
& \lambda_{1}+\lambda_{2}=a .
\end{aligned}
$$

The solution for the above system is

$$
\begin{aligned}
G_{1} & =2-a \\
G_{2} & =a-1 \\
\lambda_{1} & =2-a \\
\lambda_{2} & =2 a-2 \\
Q_{1} & =2 d(2-a) \\
Q_{2} & =2 d(a-1) \\
b_{1} & =2 d \\
b_{2} & =2 d .
\end{aligned}
$$

The average delay at equilibrium of Proportional policy is

$$
\frac{\lambda_{1} b_{1}+\lambda_{2} b_{2}}{\lambda_{1}+\lambda_{2}}=2 d
$$

\subsection{BackPressure policy}

The BackPressure policy would allocate all green time to the route that have longer queue, so at equilibrium, the queues must have the same lengths

$$
Q_{1}=Q_{2} \text {. }
$$

The equilibrium state of BackPressure policy

$$
\begin{aligned}
& b_{1}=b_{2} \\
& \frac{d Q_{1}}{d t}=0 \\
& \frac{d Q_{2}}{d t}=0 \\
& Q_{1}=Q_{2} \\
& G_{1}+G_{2}=1 \\
& \lambda_{1}+\lambda_{2}=a .
\end{aligned}
$$

The solution for the above system is

$$
\begin{aligned}
G_{1} & =2-a \\
G_{2} & =a-1 \\
\lambda_{1} & =2-a \\
\lambda_{2} & =2 a-2 \\
Q_{1} & =\frac{2 d(2-a)(a-1)}{3 a-4} \\
Q_{2} & =\frac{2 d(2-a)(a-1)}{3 a-4} \\
b_{1} & =\frac{2 d(a-1)}{3 a-4} \\
b_{2} & =\frac{2 d(a-1)}{3 a-4} .
\end{aligned}
$$

The average delay at equilibrium of BackPressure policy is

$$
\frac{\lambda_{1} b_{1}+\lambda_{2} b_{2}}{\lambda_{1}+\lambda_{2}}=\frac{2 d(a-1)}{3 a-4} .
$$

4.3. BackPressure policy with influence on users' route choice

Lets assume it is feasible to influence on users in such a way that there are more people choose route 2. It could be either increase the speed limit of route 2 or advertising a smaller free flow travel time of route 2 . Under the effect of the new control policy, let $d_{0}$, where $d_{0}<d$, be the new free flow delay perceived by users. Thus, users would choose route 1 if 
the perceived travel time on route 1 is less than the perceived travel time on route 2 , which is $\frac{Q_{1}}{G_{1}}<d_{0}+\frac{Q_{2}}{2 G_{2}}$, and choose route 2 otherwise.

The equilibrium state of BackPressure policy with influence

$$
\begin{aligned}
& \frac{Q_{1}}{G_{1}}=d_{0}+\frac{Q_{2}}{2 G_{2}} \\
& \frac{d Q_{1}}{d t}=0 \\
& \frac{d Q_{2}}{d t}=0 \\
& Q_{1}=Q_{2} \\
& G_{1}+G_{2}=1 \\
& \lambda_{1}+\lambda_{2}=a .
\end{aligned}
$$

The solution for the above system is

$$
\begin{aligned}
G_{1} & =2-a \\
G_{2} & =a-1 \\
\lambda_{1} & =2-a \\
\lambda_{2} & =2 a-2 \\
Q_{1} & =\frac{2 d_{0}(2-a)(a-1)}{3 a-4} \\
Q_{2} & =\frac{2 d_{0}(2-a)(a-1)}{3 a-4} \\
b_{1} & =\frac{2 d_{0}(a-1)}{3 a-4} \\
b_{2} & =d+\frac{d_{0}(2-a)}{3 a-4} .
\end{aligned}
$$

Note that now $b_{1} \neq b_{2}$ due to the effect influenced on users. The average delay at equilibrium is

$$
\begin{aligned}
& \frac{\lambda_{1} b_{1}+\lambda_{2} b_{2}}{\lambda_{1}+\lambda_{2}} \\
& =\frac{2}{a}(a-1)\left(d-d_{0} \frac{a-2}{3 a-4}\right) .
\end{aligned}
$$

As an example, let $d=2, d_{0}=1$, and $a=1.9$. So the average delay in the Proportional policy, BackPressure policy, and BackPressure policy with users' route choice influence are 4, 2.12, and 1.95 respectively. Clearly, for this simple network with certain parameters, it is indeed beneficial to influence on users' route choice.

\section{Numerical results - performance evaluation and comparison}

In this section we evaluate via simulation the performance of our proposed utility optimization based BackPressure (U-BP) traffic signal control and compare its performance with the $P_{0}$ policy (Smith, 2011) and the BackPressure policy (Wunderlich et al., 2008; Varaiya, 2013; Wongpiromsarn et al., 2012). The $P_{0}$ policy allocates green time proportionally with the total upstream queues of the corresponding phase, thus it will be refer to as Proportional policy in this section. The existing BackPressure policy allocates the entire green time to the phase that has the highest queue backlog differences between the upstream queues and downstream queue, and will be referred to as BackPressure policy in this section. As for our Utility optimization-based BackPressure policy, it attempts to balance between the higher weights and a larger inequality (or spatial heterogeneity) of traffic by solving a joint optimization problem using an appropriate chosen utility function. The Proportional, BackPressure, and our proposed policy schemes are briefly summarized below, respectively.

- Proportional policy (Smith, 2011)

1. At the beginning of each time slot, for each junction $j \in \mathcal{J}$, calculate the weight associated with each service phase at the junction as

$$
w_{\Psi}(Q(t))=\sum_{i \in \mathcal{I}_{j}} \psi_{i}^{j}\left(q_{i}(t)\right)
$$

2. Given these weights, within the next time slot assign a fraction of time slot to each phase $\Psi \in \mathcal{S}_{j}$ that is proportional to

$$
p_{\Psi}^{j}(t)=\frac{w_{\Psi}(Q(t))}{\sum_{\Psi \in \mathcal{S}_{j}} w_{\Psi}(Q(t))}
$$




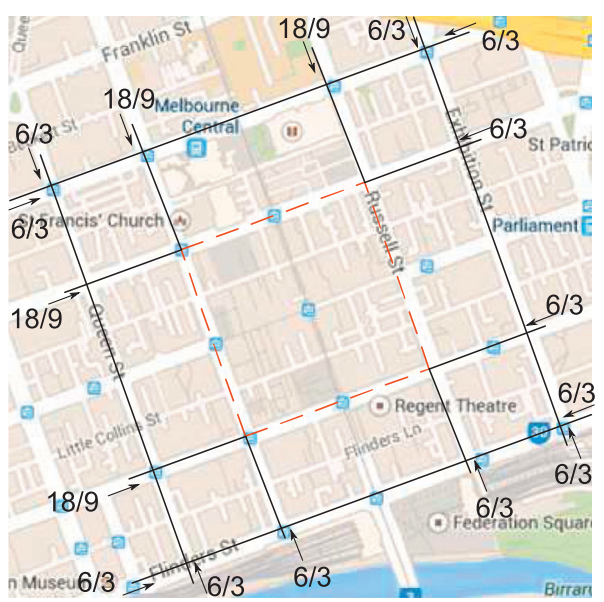

(a) Melbourne CBD.

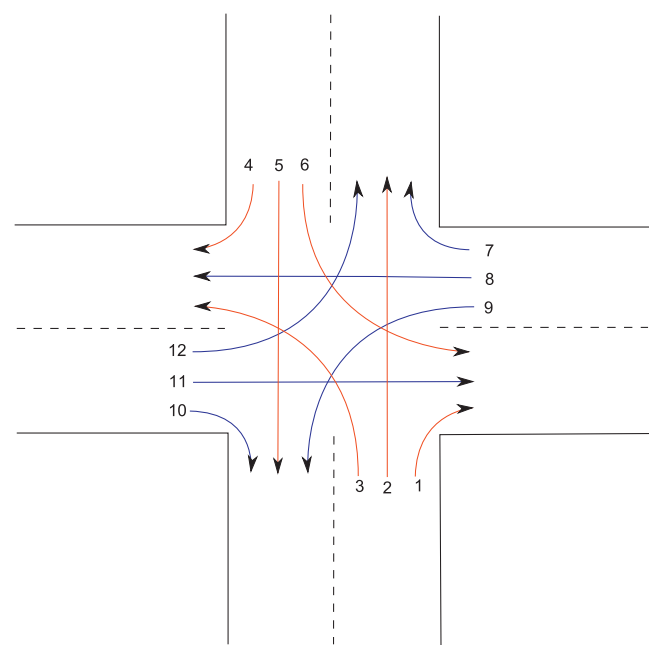

(b) Traffic movements.

Fig. 2. Network topology.

- BackPressure policy (Wunderlich et al., 2008; Varaiya, 2013; Wongpiromsarn et al., 2012):

1. At the beginning of each time slot, for each junction $j \in \mathcal{J}$, calculate the weight associated with each service phase at the junction as

$$
w_{\Psi}(Q(t))=\sum_{i \in \mathcal{I}_{j}} \psi_{i}^{j}\left(q_{i}(t)-\sum_{i^{\prime}: i^{\prime} \in \mathcal{L}} \bar{\theta}_{i i^{\prime}}(t) q_{i^{\prime}}(t)\right) .
$$

Here in this policy, $\bar{\theta}_{i i^{\prime}}$ is fixed and known priori for each of the intersections.

2. Given these weights, assign the whole service time of the next time slot to phase $\Psi^{*} \in \mathcal{S}_{j}$ where $w_{\Psi^{*}}>w_{\Psi} \forall \Psi \in \mathcal{S}_{j}$. - Utility optimization-based BackPressure (U-BP) policy proposed in this paper: Refer to Section 2.3 for details where the local Gini index function is defined as

$$
g_{j}(t+1)=\frac{1}{2 \operatorname{card}\left(\mathcal{I}_{j}\right) \sum_{i \in j} q_{i}(t+1)} \sum_{i \in j} \sum_{k \neq i, k \in j}\left|q_{i}(t+1)-q_{k}(t+1)\right|,
$$

where $\operatorname{card}(A)$ is the cardinality of a set $A$. Note that the equation (35) represents a local Gini index for junction $j$.

We remarked that the Gini index use the above has a finite order of magnitude as queue sizes grow. That is when there are relatively moderate number of cars at the junction, we expected the Gini index will impact on the control decision of the traffic light. However as injunction becomes busy it becomes increasingly pertinent with control decisions that bring the system back to stable behaviour. This is why it is important for the parameters $g_{j}$ to remain bounded. In summary, our framework allows us to balance between the BackPressure, for stability control, and the aggregate value of $g_{\text {, }}$, for the spatial homogeneity in the objective. In equation (7), this weight is set to 1 and essentially represents the trade of between the average queue length (stability) and the secondary objective associating with the $g_{j}$ function (i.e. Gini coefficient as a spatial heterogeneity indicator) where for moderate loads the Gini index will dominate ensuring a fair distribution of queue length, and for heavier traffic BackPressure will begin to take effect and ensure that system-wide stability is ensured.

\subsection{Simulation settings}

We compare the performance of the above control strategies using a section of the Melbourne CBD network that reassembles a 4-by-4 grid network topology as shown in Fig. 2(a). It consists of 16 intersections and 56 roads. For simplicity we consider all the roads to be bi-directional (i.e. a total of 112 mono-directional links) with three separate lanes in each direction assigned for the three possible traffic movements: going straight, turning left and right, respectively. Each traffic light signal at an intersection contains 2 phases, North-South(traffic movement number 1-6) and East-West(traffic movement number 7-12) (see Fig. 2(b)). The ingress queues, where vehicles enter the network, are assumed to be infinite and represented by a set of long links of approx. $1200 \mathrm{~m}$ in length. For the rest of the links inside the network, the length is set to be the same and equals to $230 \mathrm{~m}$ all of which are approximately the actual distance of the Melbourne CBD network. Since the ingress queues are large, vehicles can enter the network even when there is a heavy congestion inside the Melbourne CBD. The speed limit is set to be $30 \mathrm{kmh}$ in the inner area of the CBD (a square marked with the dash line in Fig. 2(a)), while it is set to $50 \mathrm{kmh}$ elsewhere. 
Table 2

Overall performance.

\begin{tabular}{lll}
\hline & Average travel time (second) & $\begin{array}{l}\text { Average number of route } \\
\text { advices sent per vehicle }\end{array}$ \\
\hline Proportional & 603 & NA \\
BP & 555 & NA \\
U-BP (5\%) & 520 & 2.91 \\
U-BP (10\%) & 459 & 2.94 \\
U-BP (20\%) & 383 & 2.82 \\
U-BP (30\%) & 369 & 2.75 \\
U-BP (50\%) & 388 & 2.93 \\
U-BP (100\%) & 507 & 3.42 \\
\hline
\end{tabular}

The demand is modeled by two consecutive pairs of a $30 \mathrm{~min}$. peak period follows by a $30 \mathrm{~min}$. off-peak period. The main traffic comes from both the West and North and moves toward the East and South sides, respectively; while the secondary traffic travels on the reverse direction (i.e. East-West and South-North directions). The demand of the main traffic are 18 and 9 cars per minutes during the peak and off-peak period, respectively. The demand of the secondary traffic is set to be one third of the main traffic (i.e. 6 and 3 cars per minutes for peak and off-peak periods) (see Fig. 2(a)). The traffic light cycle and system unit time step is set to be one minute (i.e. $60 \mathrm{~s}$ ) where we ignore the switching times or transition between phases in all the control schemes in our study. This overhead can be incorporated into the simulation by extending the phase times. Nevertheless, the qualitative insights gained in this section would not change by that extension.

Route guidance (via e.g. advanced traveler information system, ATIS) is implemented in the U-BP scheme so that the queue lengths in each lane can be approximately maintained to be proportional to the optimal turning fractions at an individual intersection. These turning fractions are resulted from distributively solving the problem in (7) using the local utility function in (35). In the simulation, however, individual vehicle will either follow the route guidance advice, or keep its original route according to an independent compliance probability $p(0 \leq p \leq 1)$. If the vehicle indeed chooses to comply with the advice, it will join the appropriate lane in order to make the advised turn at the intersection and then follow the new shortest path to its destination afterward. Regardless of the drivers' decision, each vehicle only receives at most one route guidance advice per link. In certain scenarios where it is impossible to reach the destination by following the advice (e.g. at the edges of the network) or it is too close to the intersection where lane changing is prohibited for safety reason, vehicle will ignore the advice and follows its current shortest path to the destination.

Below we will use an open source microscopic simulation package SUMO (Simulation of Urban MObility) (SUMO, 2013) to study the performance of the control strategies and obtain the results. During simulation, the exact queue lengths are observed and used to make control decision in various policies. In particular, the optimal control decisions are solved using Matlab (MATLAB, 2013) and its optimization tools based on the actual control algorithm, and results are then fed back into the SUMO simulation in every time step (or traffic light cycle).

Results are given in terms of the network throughput, the total number of vehicles in the network over time, and the spatial heterogeneity of traffic density in terms of the Gini index after several long simulation runs using the different control schemes.

\subsection{Results and discussions}

Results for the average travel time and the average number of advices per vehicle over its entire journey using the Proportional policy, the original BackPressure policy (referred to as BP), and the proposed utility optimization-based BackPressure policy (referred to as U-BP) with different compliance percentages (in brackets) are shown in Table 2.

Observe that the U-BP scheme yields a lower average travel time even when only a small percentage of vehicles complying to the route guidance advice, e.g. the travel time of U-BP (5\%) is better than both that of the proportional policy and original BP policy. Furthermore, the average travel time decreases as the compliance rate increases up to $30 \%$ of user compliance where the trend reverses. It is because the route guidance (by way of the controller) tends to divert traffic to avoid the congested inner area thus improves the network overall travel time. However, when there are too many vehicles follow the route guidance advice (e.g. in the cases of $50 \%$ and $100 \%$ compliance probability), the overall benefits are out weighted by the lost in travel time over the longer paths, and thus the overall network performance decreases. This is inherently the nature of distributed approaches where the route guidance advice is only based on local information. The route advices in this case are solely for the purpose of local performance which might diver traffic to longer paths resulting in the degradation of the overall network performance. Our results suggest that it might not be wise to make the route guidance information accessible for every vehicle. This is in line with empirical observations that have been reported in the literature (Emmerink et al., 1995; Tsubota et al., 2013). Nevertheless, the proposed policy show superior performance compared with other distributed policies without turning advice. 
(a)

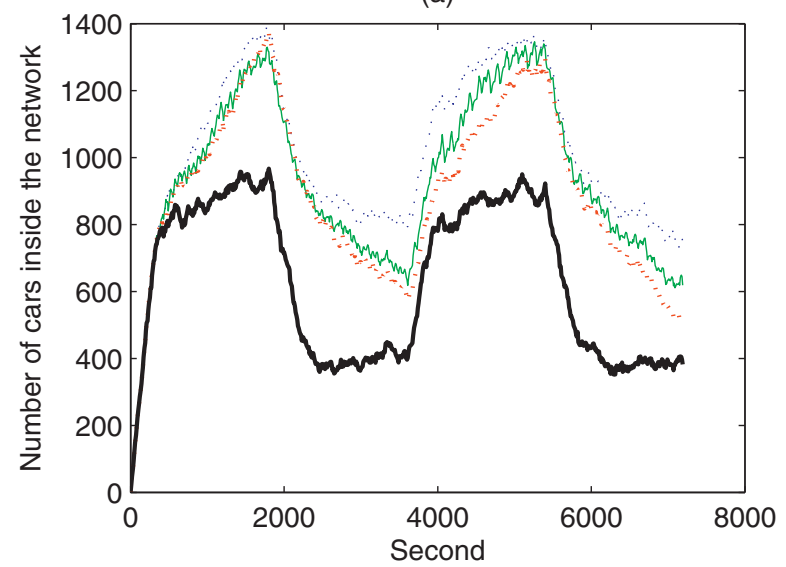

(c)

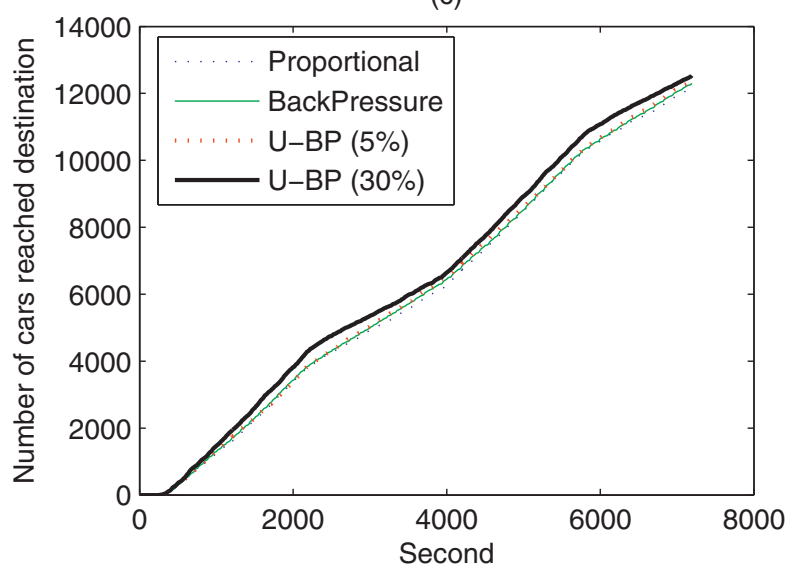

(b)

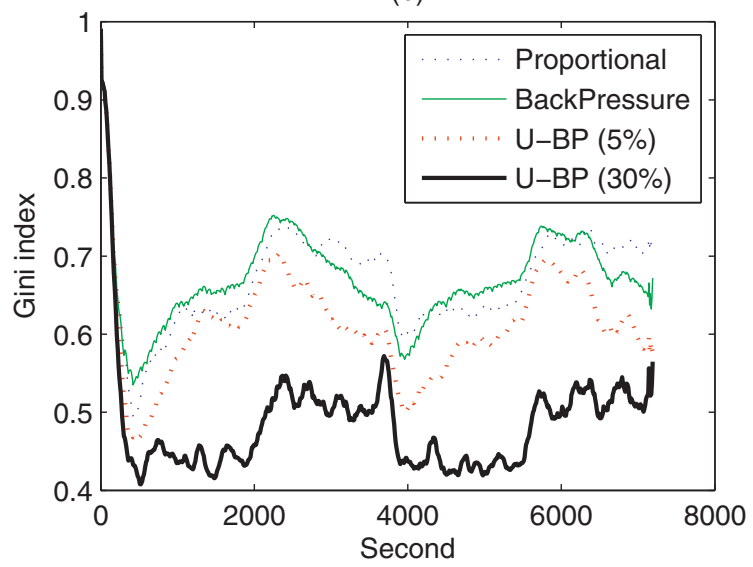

(d)

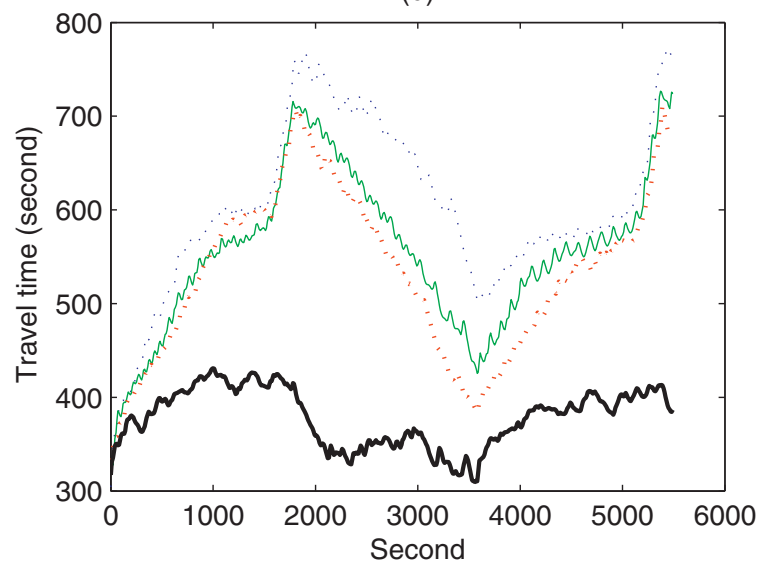

Fig. 3. Simulation results.

The number of average advice received by a vehicle varies depend on the compliance probability (see Table 2). Generally, the controller is prone to send more advices with low compliance probability because when a vehicle does not comply, another advice will be sent to different vehicle in order to achieve the desired turning fractions. The travel routes also have a role on the number of advices as vehicle on longer route is more likely to receive more advices. This explains why the lowest number of advices occurs in the case of optimal travel time.

Further analysis of the level of congestion, traffic spatial heterogeneity (in terms of the Gini index), network throughput, and network travel time using the Proportional, the BackPressure and U-BP (with 5\% and 30\% compliance probability) schemes are shown in Fig. 3(a-d) respectively. Observe that the U-BP (30\%) control yields a significant lower number of total vehicles present in the network (Fig. 3(a)) and thus results in a higher number of vehicles reaching their destination (i.e. increased network throughput, Fig. 3(c)) during the simulation. This is explained by the fact that in the U-BP control scheme when the network is congested, some traffic will be diverted from the inner CBD area to outer connected roads of the considered network. This is a result of solving the optimization problem aiming to minimize the traffic spatial heterogeneity (Fig. 3(b)) in the network through the use of the Gini utility function. Furthermore, note that the average travel time of vehicles through the network is also improved significantly (see Fig. 3(d)).

Fig. 4 shows the congested links in the network over time for each of the policies. A link is said to be congested at a certain time if its queue length is more than $75 \%$ of the maximum number of cars the link can physically hold. The figure shows that congestion almost non-exists during the low demand period for the U-BP (30\%) scheme while persists throughout the entire simulation for the other schemes. It implies that the U-BP (30\%) scheme is able to serve more vehicles during the peak period, and resolves congestion in the network quicker resulting in a higher network throughput in overall.

In summary, our numerical results show that the proposed utility optimization based BackPressure (U-BP) can greatly improve on the network spatial heterogeneity while retains the important stability feature and good performance of the original BackPressure signal control strategy. 
(a)Congestion of the Proportional policy
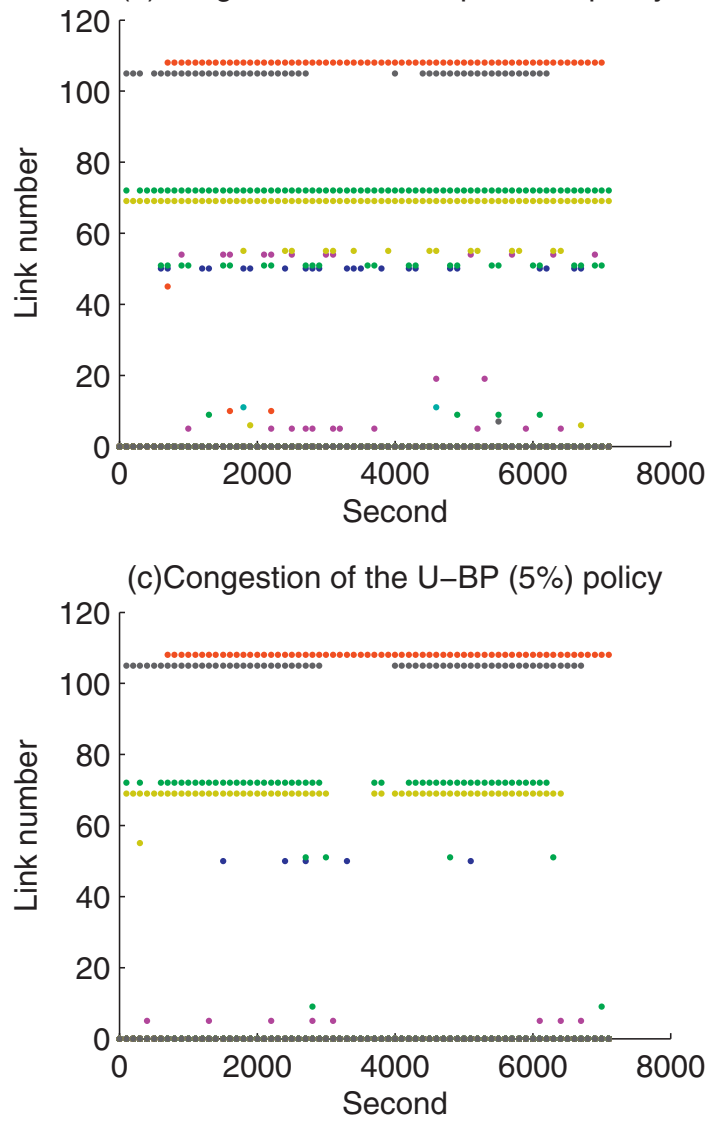

(b)Congestion of the BackPressure policy

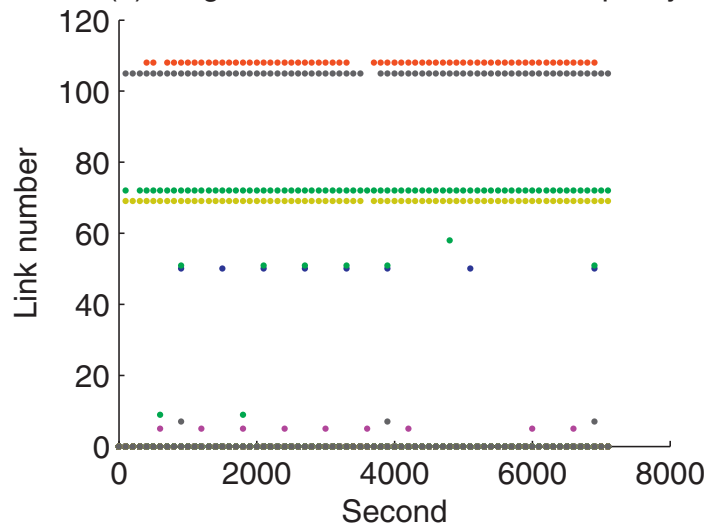

(d)Congestion of the U-BP (30\%) policy

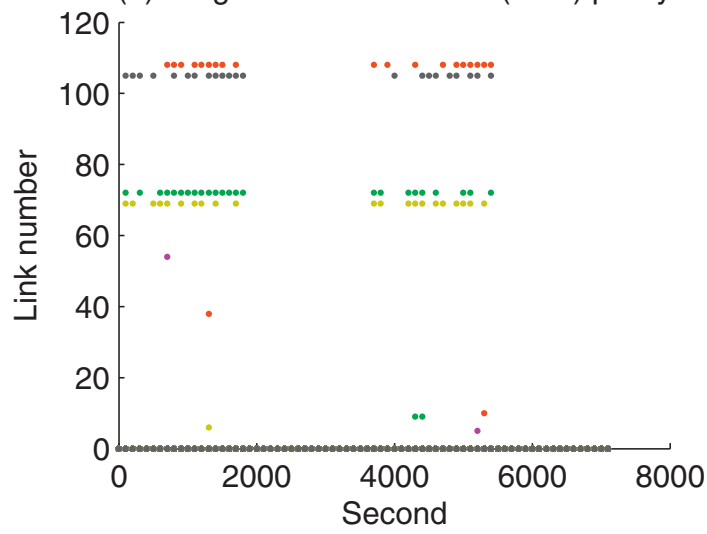

Fig. 4. Congestion of each control scheme.

\section{Conclusion}

In this paper we proposed a novel distributed signal control strategy based on the BackPressure strategy. This strategy does not require any prior knowledge of the traffic demand and only requires information (i.e. queue size) that is local to the intersection. In contrast to previous BackPressure-based strategies in which large queues tend to build up forming unnecessary congestion, our scheme maximizes the road network throughput while minimizing the risk of congestion forming through a novel utility optimization mathematical framework.

Given the proposed strategy, we have formally proven the stability of the utility optimization-based BackPressure signal control and showed through simulation and numerical results that the new policy outperforms the original BackPressurebased distributed control scheme both in terms of network throughput and other congestion measures such as travel time. This significant outcome has been achieved by applying the Gini-index as an utility function in our optimization framework to optimally obtain the signal phasings and to proactively influence the drivers' route choice behavior (via route guidance advice) and avoid the congestion onset. Our results demonstrated that the network performance improves significantly even with limited user compliance, and a relatively small percentage of compliance (e.g. 10\%) is enough to gain significant benefits (e.g. $17 \%$ reduction in average travel time).

\section{Acknowledgment}

This work was supported by the Australian Research Council(ARC) Future Fellowships grant FT120100723.

\section{Appendix A. Proof of Theorem 1}

First let us define the term

$$
\Xi(t)=\sum_{i \in \mathcal{I}} q_{i}(t)\left(\sum_{\Psi \in \mathcal{S}_{j}, j: i \in \mathcal{I}_{j}} \psi_{i} p_{\Psi}^{j}(t)-\sum_{i^{\prime}: i^{\prime} i \in \mathcal{L}} \sum_{\Psi \in \mathcal{S}_{j^{\prime}}, j^{\prime}: i^{\prime} \in \mathcal{I}_{j^{\prime}}} \psi_{i^{\prime}} p_{\Psi}^{j}(t) \bar{\theta}_{i^{\prime} i}(t)\right) .
$$


In our first lemma, we argue that it can be rearranged to give the second part of the object function in (7).

Lemma 1. The following equality holds

$$
\Xi(t)=\sum_{k \in \mathcal{J}} \sum_{\Psi \in \mathcal{S}_{k}} p_{\Psi}^{k}(t) \sum_{i \in \mathcal{I}_{k}} \psi_{i}\left(q_{i}(t)-\sum_{i^{\prime}: i^{\prime} \in \mathcal{L}} q_{i^{\prime}}(t) \bar{\theta}_{i i^{\prime}}(t)\right) .
$$

Proof. The proof is straight forward by rearranging the sum:

$$
\begin{aligned}
\sum_{i \in \mathcal{I}} q_{i}(t) & \left(\sum_{\Psi \in \mathcal{S}_{j}, j: i \in \mathcal{I}_{j}} \psi_{i} p_{\Psi}^{j}(t)-\sum_{i^{\prime}: i^{\prime} i \in \mathcal{L}} \sum_{\Psi \in \mathcal{S}_{j^{\prime}} j^{\prime}: i^{\prime} \in \mathcal{I}_{j^{\prime}}} \psi_{i^{\prime}} p_{\Psi}^{j}(t) \bar{\theta}_{i^{\prime}}(t)\right) \\
= & \sum_{i \in \mathcal{I}} \sum_{\Psi \in \mathcal{S}_{j}, j: i \in \mathcal{I}_{j}} q_{i}(t) \psi_{i} p_{\Psi}^{j}(t)-\sum_{i \in \mathcal{I}} \sum_{i^{\prime}: i^{\prime} i \in \mathcal{L}} \sum_{\Psi \in \mathcal{S}_{j^{\prime}}, j^{\prime}: i^{\prime} \in \mathcal{I}_{j^{\prime}}} q_{i}(t) \psi_{i^{\prime}} p_{\Psi}^{j}(t) \bar{\theta}_{i^{\prime} i}(t) \\
= & \sum_{k \in \mathcal{J}} \sum_{\Psi \in \mathcal{S}_{k}} p_{\Psi}^{k}(t) \sum_{i \in \mathcal{I}_{k}} \psi_{i}\left(q_{i}(t)-\sum_{i^{\prime}: i^{\prime} \in \mathcal{L}} q_{i^{\prime}}(t) \bar{\theta}_{i i^{\prime}}(t)\right)
\end{aligned}
$$

Secondly, we prove the a bound on the sample paths of the queue size process using our assumptions in Section 3.1.

Lemma 2. There exists a constant $K \geq 0$ such that the queue size process obeys the bound

$$
\frac{1}{2} q_{i}(t+1)^{2}-\frac{1}{2} q_{i}(t)^{2} \leq q_{i}(t)\left(a_{i}(t)-s_{i}(t)+\sum_{i^{\prime}: i^{\prime} \in \mathcal{L}} s_{i^{\prime}}(t) \theta_{i^{\prime}}(t)\right)+K
$$

Proof. From the queue length process equation, we have

$$
\begin{aligned}
& \frac{1}{2} q_{i}(t+1)^{2}-\frac{1}{2} q_{i}(t)^{2} \\
& =\frac{1}{2}\left(a_{i}(t)-f_{i}(t)+\sum_{i^{\prime}: i^{\prime} \in \mathcal{L}} f_{i^{\prime}}(t) \theta_{i^{\prime}}(t)\right)^{2}+q_{i}(t)\left(a_{i}(t)-f_{i}(t)+\sum_{i^{\prime} i^{\prime} i^{\prime} \in \mathcal{L}} f_{i^{\prime}}(t) \theta_{i^{\prime} i}(t)\right) \\
& =\frac{1}{2}\left(a_{i}(t)+\sum_{i^{\prime} i_{i}^{\prime} \in \mathcal{L}} f_{i^{\prime}}(t) \theta_{i^{\prime} i}(t)\right)^{2}+\frac{1}{2}\left(f_{i}(t)\right)^{2}-f_{i}(t)\left(a_{i}(t)+\sum_{i^{\prime}: i^{\prime} \in \mathcal{L}} f_{i^{\prime}}(t) \theta_{i^{\prime} i}(t)\right) \\
& +q_{i}(t)\left(a_{i}(t)-f_{i}(t)+\sum_{i^{\prime}: i^{\prime} i \in \mathcal{L}} f_{i^{\prime}}(t) \theta_{i^{\prime} i}(t)\right) \\
& \leq \frac{1}{2}\left(A_{\max }+D_{\max } S_{\max }\right)^{2}+\frac{1}{2} S_{\max }^{2}+q_{i}(t)\left(a_{i}(t)-\min \left(s_{i}(t), q_{i}(t)\right)+\sum_{i^{\prime}: i^{\prime} i \in \mathcal{L}} s_{i^{\prime}}(t) \theta_{i^{\prime} i}(t)\right)
\end{aligned}
$$

In the inequality above, we not that the first two terms of expression (A.4) are bounded above by constants and the third term is negative.

Now if $s_{i}(t) \geq q_{i}(t)$ then, since

$$
-q_{i} \min \left(q_{i}, s_{i}\right)=-q_{i}^{2} \leq-q_{i} s_{i}+s_{i}^{2},
$$

we have that

$$
\frac{1}{2} q_{i}(t+1)^{2}-\frac{1}{2} q_{i}(t)^{2} \leq \frac{1}{2}\left(A_{\max }+D_{\max } S_{\max }\right)^{2}+\frac{1}{2} S_{\max }^{2}+q_{i}(t)\left(a_{i}(t)-s_{i}(t)+\sum_{i^{\prime} i^{\prime} i \in \mathcal{L}} s_{i^{\prime}}(t) \theta_{i^{\prime} i}(t)\right)+S_{\max }^{2} .
$$

If $s_{i}(t)<q_{i}(t)$ then

$$
\frac{1}{2} q_{i}(t+1)^{2}-\frac{1}{2} q_{i}(t)^{2} \leq \frac{1}{2}\left(A_{\max }+D_{\max } S_{\max }\right)^{2}+\frac{1}{2} S_{\max }^{2}+q_{i}(t)\left(a_{i}(t)-s_{i}(t)+\sum_{i^{\prime} i^{\prime} i^{\prime} \in \mathcal{L}} s_{i^{\prime}}(t) \theta_{i^{\prime} i}(t)\right) .
$$

We see that in both cases, $s_{i}(t) \geq q_{i}(t)$ or $s_{i}(t)<q_{i}(t)$, we have the required bound with

$$
K=\frac{1}{2}\left(A_{\max }+D_{\max } S_{\max }\right)^{2}+\frac{3 S_{\max }^{2}}{2}
$$


In our next lemma we prove a bound on the change in the (euclidean) distance of our queue sizes from zero.

Lemma 3. With the same constant $K$ it holds that

$$
\sum_{i \in \mathcal{I}} \mathbb{E}\left[\frac{1}{2} q_{i}(t+1)^{2}-\frac{1}{2} q_{i}(t)^{2}\right] \leq \mathbb{E}\left[\sum_{i \in \mathcal{I}} q_{i}(t) \bar{a}_{i}-\Xi(t)\right]+|\mathcal{I}| K .
$$

Proof. In order to prove Lemma 3 we are going to use Lemma 2 as well as the following equalities.

$$
\mathbb{E}\left[q_{i}(t) a_{i}(t)\right]=\mathbb{E}\left[q_{i}(t) \bar{a}_{i}\right]
$$

and

$$
\begin{aligned}
& \mathbb{E}\left[q_{i}(t)\left(-s_{i}(t)+\sum_{i^{\prime}: i^{\prime} i \in \mathcal{L}} s_{i^{\prime}}(t) \theta_{i^{\prime} i}(t)\right)\right] \\
& \quad=\mathbb{E}\left[q_{i}(t)\left(-\sum_{\Psi \in \mathcal{S}_{j}, j: i \in \mathcal{I}_{j}} \psi_{i} p_{\Psi}^{j}(t)+\sum_{i^{\prime}: i^{\prime} \in \mathcal{L}} \sum_{\Psi \in \mathcal{S}_{j^{\prime}}, j^{\prime}: i^{\prime} \in \mathcal{I}_{j^{\prime}}} \psi_{i^{\prime}} p_{\Psi}^{j}(t) \bar{\theta}_{i^{\prime} i}(t)\right)\right] .
\end{aligned}
$$

The above equality holds by (2) and (4).

Thus we have

$$
\begin{array}{rl}
\sum_{i \in \mathcal{I}} & \mathbb{E} \\
\quad \leq \sum_{i \in \mathcal{I}} \mathbb{E}\left[q_{i}(t)\left(a_{i}(t)-s_{i}(t)+\sum_{i^{\prime} i^{\prime} i^{\prime} \in \mathcal{L}} s_{i^{\prime}}(t) \theta_{i^{\prime} i}(t)\right)+K\right] \\
\quad=\mathbb{E}\left[\sum_{i \in \mathcal{I}} q_{i}(t) \bar{a}_{i}-\Xi(t)\right]+|\mathcal{I}| K .
\end{array}
$$

As the next step, we define stationary policies to further elaborate our bounds. Stationary policies are the set of policies that allocate green time randomly each time slot as independent identical distributed random variables. For example, a fixed policy that allocates constant green time duration is a stationary policy. Now assume that the arrival process is known in advance and there is a positive value $\epsilon$ such that $(\bar{A}+\epsilon) \in \mathcal{A}$. Then there exists a set of departure rates $\bar{F}$, green time proportions $\bar{P} \in \Pi$, and the turning fractions $\bar{\Theta} \in \mathcal{H}$ satisfying the constraints (10) and (11). Assume further that in every intersection it is possible to assign the green time proportions that the offered service rates equal $\bar{F}$.

Now define a stationary policy, which at every time slot allocates constant green time proportions and constant the turning fractions such that

$$
s_{i}^{S T A T}(t)=\sum_{\Psi \in \mathcal{S}_{j}, j: i \in \mathcal{I}_{j}} \psi_{i} p_{\Psi}^{j}(t)=\bar{f}_{i} \quad \forall i \in \mathcal{I}, \forall t \in \mathbb{N}_{0}
$$

and

$$
\theta_{i i^{\prime}}^{\text {STAT }}(t)=\bar{\theta}_{i i^{\prime}} \forall i, i^{\prime} \in \mathcal{I}, \forall t \in \mathbb{N}_{0} .
$$

According to (10) for any time slot $t$, the stationary policy achieves the following bound

$$
s_{i}^{S T A T}(t)-\sum_{i^{\prime}: i^{\prime} i \in \mathcal{L}} s_{i^{\prime}}^{S T A T}(t) \theta_{i^{\prime} i}^{S T A T}(t)-\bar{a}_{i} \geq \epsilon .
$$

Substitute (A.7) in (A.8) to get

$$
\sum_{\Psi \in \mathcal{S}_{j}, j: i \in \mathcal{I}_{j}} \psi_{i} p_{\Psi}^{j, S T A T}(t)-\sum_{i^{\prime}: i^{\prime} i \in \mathcal{L}} \sum_{\Psi \in \mathcal{S}_{j^{\prime}}, j^{\prime}: i^{\prime} \in \mathcal{I}_{j^{\prime}}} \psi_{i^{\prime}} p_{\Psi}^{j, S T A T}(t) \theta_{i^{\prime} i}^{\text {STAT }}(t)-\bar{a}_{i} \geq \epsilon .
$$

If we multiply by $-\sum_{i \in \mathcal{I}} q_{i}(t)$ and use the definition in (A.1), we get

$$
\sum_{i \in \mathcal{I}} q_{i}(t) \bar{a}_{i}-\Xi^{S T A T}(t) \leq-\epsilon \sum_{i \in \mathcal{I}} q_{i}(t) .
$$

This bound helps us in proving the following lemma. 
Lemma 4. For the proposed BackPressure policy the following bound holds for the queue sizes at any given time slot $t$,

$$
\sum_{i \in \mathcal{I}} \mathbb{E}\left[\frac{1}{2} q_{i}(t+1)^{2}-\frac{1}{2} q_{i}(t)^{2}\right] \leq|\mathcal{J}| G+|\mathcal{I}| K-\epsilon \mathbb{E}\left[\sum_{i \in \mathcal{I}} q_{i}(t)\right] .
$$

Proof. We provide an argument by comparing the BackPressure policy and the stationary policy. First we show that the proposed BackPressure policy minimizes $\left(\sum_{j \in \mathcal{J}} g_{j}(t)-\Xi(t)\right)$ over all possible policies including the stationary policy.

$$
\begin{aligned}
& \sum_{j \in \mathcal{J}} \min _{P_{j}(t), \bar{\Theta}_{j}(t)} g_{j}(t)-\sum_{\Psi \in \mathcal{S}_{j}} p_{\Psi}^{j}(t) w_{\Psi}(Q(t)) \\
& =\min _{P_{j}(t), \bar{\Theta}_{j}(t)} \sum_{j \in \mathcal{J}} g_{j}(t)-\sum_{j \in \mathcal{J}} \sum_{\Psi \in \mathcal{S}_{j}} p_{\Psi}^{j}(t) w_{\Psi}(Q(t)) \\
& =\min _{P_{j}(t), \bar{\Theta}_{j}(t)} \sum_{j \in \mathcal{J}} g_{j}(t)-\Xi(t) .
\end{aligned}
$$

Note that the equality occurs because $P_{j}$ and $\bar{\Theta}_{j}$ are disjoint. Thus, it holds that

$$
\sum_{j \in \mathcal{J}} g_{j}^{B P}(t)-\Xi^{B P}(t) \leq \sum_{j \in \mathcal{J}} g_{j}^{S T A T}(t)-\Xi^{S T A T}(t)
$$

Now by reordering the term and considering the fact that the utility function is bounded, we get

$$
-\Xi^{B P}(t) \leq \sum_{j \in \mathcal{J}} g_{j}^{S T A T}(t)-g_{j}^{B P}(t)-\Xi^{S T A T}(t) \leq|\mathcal{J}| G-\Xi^{S T A T}(t) .
$$

Thus by (A.9),

$$
\begin{aligned}
& \sum_{i \in \mathcal{I}} q_{i}(t) \bar{a}_{i}(t)-\Xi^{B P}(t) \\
& \quad \leq|\mathcal{J}| G+\sum_{i \in \mathcal{I}} q_{i}(t) \bar{a}_{i}(t)-\Xi^{S T A T}(t) \\
& \quad \leq|\mathcal{J}| G-\epsilon \sum_{i \in \mathcal{I}} q_{i}(t) .
\end{aligned}
$$

Now if we finish the proof by combining (A.6) with (A.11) as

$$
\begin{array}{rl}
\sum_{i \in \mathcal{I}} & \mathbb{E}\left[\frac{1}{2} q_{i}(t+1)^{2}-\frac{1}{2} q_{i}(t)^{2}\right] \\
\leq & \mathbb{E}\left[\sum_{i \in \mathcal{I}} q_{i}(t) \bar{a}_{i}-\Xi^{B P}(t)\right]+|\mathcal{I}| K \\
\leq|\mathcal{J}| G+|\mathcal{I}| K-\epsilon \mathbb{E}\left[\sum_{i \in \mathcal{I}} q_{i}(t)\right] .
\end{array}
$$

The following proposition finalizes the proof.

Proposition 2. If there exists positive constant $C$ and $\delta$ such that for all time slot $t$, the Lyapunov drift satisfies

$$
\sum_{i \in \mathcal{I}}\left(\mathbb{E}\left[q_{i}(t+1)\right]^{2}-\mathbb{E}\left[q_{i}(t)\right]^{2}\right) \leq C-\delta \sum_{i \in \mathcal{I}} \mathbb{E}\left[q_{i}(t)\right],
$$

then the network is stable by the definition given in (9). 
Proof. We create a telescoping series by summing over $t$ from $t_{0}$ to $T$ :

$$
\sum_{i \in \mathcal{I}}\left(\mathbb{E}\left[q_{i}(t+T)\right]^{2}-\mathbb{E}\left[q_{i}\left(t_{0}\right)\right]^{2}\right) \leq T C-\delta \sum_{t=t_{0}}^{T-1} \sum_{i \in \mathcal{I}} \mathbb{E}\left[q_{i}(t)\right] .
$$

Taking the limit $T \rightarrow \infty$ :

$$
\begin{aligned}
& \lim _{T \rightarrow \infty} \frac{1}{T} \sum_{t=t_{0}}^{T-1} \sum_{i \in \mathcal{I}} \mathbb{E}\left[q_{i}(t)\right] \leq \frac{C}{\delta}+\lim _{T \rightarrow \infty} \frac{1}{T \delta} \sum_{i \in \mathcal{I}} \mathbb{E}\left[q_{i}\left(t_{0}\right)\right]^{2} . \\
& \lim _{T \rightarrow \infty} \frac{1}{T} \sum_{t=t_{0}}^{T-1} \sum_{i \in \mathcal{I}} \mathbb{E}\left[q_{i}(t)\right] \leq \frac{C}{\delta} .
\end{aligned}
$$

Which is the stability condition described in (9).

Now if we consider the bound given in Lemma 4, it is clea $r$ that by taking $C=|\mathcal{I}| K|\mathcal{J}| G$ and $\delta=\epsilon$, the condition in (A.12) is fulfilled. Thus we have proven stability for the network.

\section{References}

Ahmed, N.U., Ahmed, N.U., 2006. Dynamic Systems and Control with Applications. World Scientific.

Allsop, R.E., 1974. Some possibilities for using traffic control to influence trip distribution and route choice. In: Transportation and Traffic Theory, Proceedings, 6.

Bui, L., Srikant, R., Stolyar, A., 2009. Novel architectures and algorithms for delay reduction in back-pressure scheduling and routing. In: INFOCOM 2009. IEEE, pp. 2936-2940.

Chen, O.J., Ben-Akiva, M.E., 1998. Game-theoretic formulations of interaction between dynamic traffic control and dynamic traffic assignment. Transp. Res. Rec. 1617 (1), 179-188.

Dujardin, Y., Boillot, F., Vanderpooten, D., Vinant, P., 2011. Multiobjective and multimodal adaptive traffic light control on single junctions. In: ITSC2011. IEEE, pp. 1361-1368.

Emmerink, R.H., Axhausen, K.W., Nijkamp, P., Rietveld, P., 1995. The potential of information provision in a simulated road transport network with non-recurrent congestion. Transp. Res. Part C 3 (5), 293-309.

Fhwa, 2016. Congestion reduction toolbox: traffic signal timing. URL www.fhwa.dot.gov/congestion/toolbox/service.htm.

Foster, J., Sen, A., 1973. On Economic Inequality. Oxford University Press.

Gartner, N.H., 1983. Opac: A demand-responsive strategy for traffic signal control. Transp. Res. Rec. 906, 75-81.

Gartner, N.H., Little, J.D., Gabbay, H., 1975. Optimization of traffic signal settings by mixed-integer linear programming: Part I: the network coordination problem. Transp. Sci. 9 (4), 321-343.

Gartner, N.H., Pooran, F.J., Andrews, C.M., 2001. Implementation of the opac adaptive control strategy in a traffic signal network. In: Proceeding of 4th IEEE Conference on Intelligent Transportation Systems, pp. 197-202.

J. de Gier, T.M.G., Rojas, O., 2011. Traffic flow on realistic road networks with adaptive traffic lights. J. Stat. Mech.

Groot, N., De Schutter, B., Hellendoorn, H., 2015. Toward system-optimal routing in traffic networks: a reverse Stackelberg game approach. IEEE Trans. Intell. Transp. Syst. 16 (1), 29-40.

Hamilton, A., Waterson, B., Cherrett, T., Robinson, A., Snell, I., 2013. The evolution of urban traffic control: changing policy and technology. Transp. Planning Technol. 36 (1), 24-43.

Hoogendoorn, S., Knoop, V., Lint, H.V., Vu, H., 2013. Generalized network fundamental diagram. In: Conference on Traffic and Granular Flow, Forschungszentrum Jlich, Germany.

Hunt, P.B., Robertson, D.I., Bretherton, R.D., Winton, R.I., 1981. SCOOT-a Traffic Responsive Method of Coordinating Signals. Technical Report.

Karoonsoontawong, A., Waller, S.T., 2010. Integrated network capacity expansion and traffic signal optimization problem: robust bi-level dynamic formulation. Netw. Spatial Econ. 10 (4), 525-550.

Lämmer, S., Helbing, D., 2008. Self-control of traffic lights and vehicle flows in urban road networks. J. Stat. Mech. 2008 (04), P04019.

Lämmer, S., Helbing, D., 2010. Self-stabilizing decentralized signal control of realistic, saturated network traffic. Santa Fe Institute.

Le, T., Kovács, P., Walton, N., Vu, H.L., Andrew, L.L., Hoogendoorn, S.S., 2015. Decentralized signal control for urban road networks. Transp. Res. Part C 58, 431-450.

Le, T., Vu, H.L., Nazarathy, Y., Vo, Q.B., Hoogendoorn, S., 2013. Linear-quadratic model predictive control for urban traffic networks. Transp. Res. Part C 36, 498-512.

Lowrie, P.R., 1982. The Sydney coordinated adaptive traffic system-principles, methodology, algorithms. In: International Conference on Road Traffic Signalling. 207

MATLAB, 2013. Matlab home page. URL http://www.mathworks.com.au/products/matlab/.

Mirchandani, P., Head, L., 2001. Rhodes: a real-time traffic signal control system: architecture, algorithms, and analysis. Transp. Res. Part C 9 (6), 415-432.

Papageorgiou, M., Diakaki, C., Dinopoulou, V., Kotsialos, A., Wang, Y., 2003. Review of road traffic control strategies. In: Proceedings of the IEEE, 91.

Rinaldi, M., Tampère, C.M., 2015. An extended coordinate descent method for distributed anticipatory network traffic control. Transp. Res. Part B 80, $107-131$.

Shu, L., Schutter, B.D., Xi, Y., Hellendoorn, H., 2011. Fast model predictive control for urban road networks via MILP. IEEE Trans. Intell. Transp. Syst. 12 (3), 846-856. doi:10.1109/TITS.2011.2114652.

Smith, M., 1980. A local traffic control policy which automatically maximises the overall travel capacity of an urban road network. Traffic Eng. Control 21 (6), 298-302.

Smith, M., 2011. Dynamics of route choice and signal control in capacitated networks. J. Choice Model. 4 (3), 30-51.

Smith, M.J., Liu, R., Mounce, R., 2015. Traffic control and route choice: capacity maximisation and stability. Transp. Res. Part B 81, 863-885.

Stolyar, A.L., 2011. Large number of queues in tandem: scaling properties under back-pressure algorithm. Queueing Syst. 67, $111-126$.

SUMO, 2013. Sumo home page. URL http://sumo-sim.org/.

Taale, H., 2008. Integrated Anticipatory Control of Road Networks: A Game-Theoretical Approach. TU Delft, Delft University of Technology.

Tassiulas, L., Ephremides, A., 1992. Stability properties of constrained queueing systems and scheduling policies for maximum throughput in multihop radio networks. IEEE Trans. Autom. Control 37 (12), 1936-1948.

Tettamanti, T., Varga, I., 2010. Distributed traffic control system based on model predictive control. Periodica Polytechnica ser. Civil. Eng. 54 (1), 3-9.

Tsubota, T., Bhaskar, A., Chung, E., 2013. Information provision and network performance represented by macroscopic fundamental diagram. In: Transportation Research Board 92nd Annual Meeting Proceedings. Transportation Research Board. 
Ukkusuri, S., Doan, K., Aziz, H.A., 2013. A bi-level formulation for the combined dynamic equilibrium based traffic signal control. In: Procedia-Social and Behavioral Sciences, 80, pp. 729-752.

Varaiya, P., 2013. The max-pressure controller for arbitrary networks of signalized intersections. In: Advances in Dynamic Network Modeling in Complex Transportation Systems. Springer, pp. 27-66.

Wongpiromsarn, T., Uthaicharoenpong, T., Wang, Y., Frazzoli, E., Wang, D., 2012. Distributed traffic signal control for maximum network throughput. In: IEEE Conference on Intelligent Transportation Systems (ITSC). IEEE, pp. 588-595.

Wunderlich, R., Liu, C., Elhanany, I., Urbanik, T., 2008. A novel signal-scheduling algorithm with quality-of-service provisioning for an isolated intersection. IEEE Trans. Intell. Transp. Syst. 9 (3), 536-547.

Zaidi, A.A., Kulcsár, B., Wymeersch, H., 2016. Back-pressure traffic signal control with fixed and adaptive routing for urban vehicular networks. IEEE Trans. Intell. Transp. Syst. 17 (8), 2134-2143.

van Zuylen, H., Taale, H., 2000. Anticipatory optimization of traffic control. Transp. Res. Rec. (1725) $109-115$. 